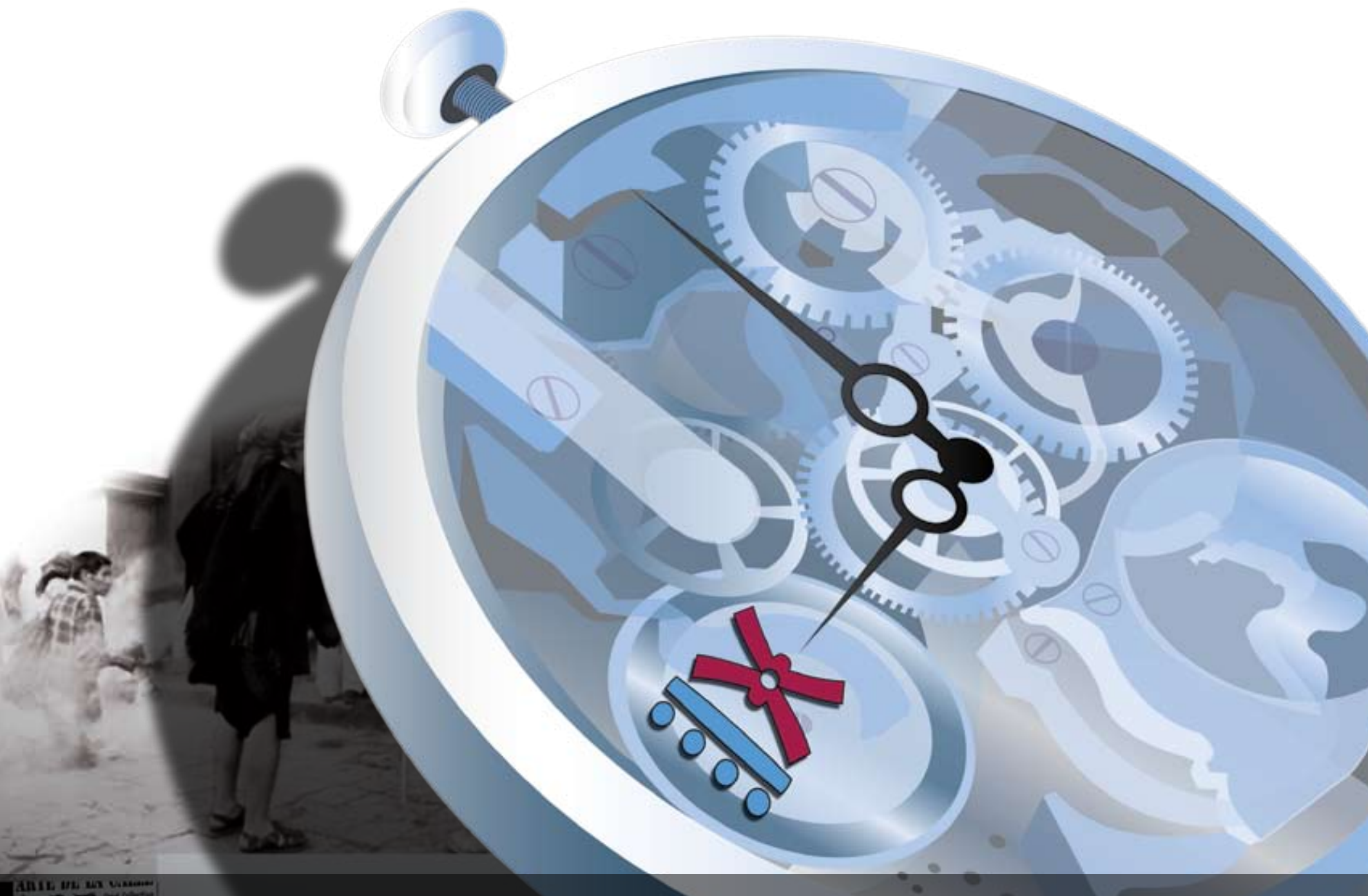

$\lambda 9^{\circ}$ C O N RESO Q 2. CENTROAMERICANO DE HISTORIA

ISSN 1409-469X

Fecha de recepción: 15 de mayo 2008 Fecha de aceptación: 30 de mayo 2008

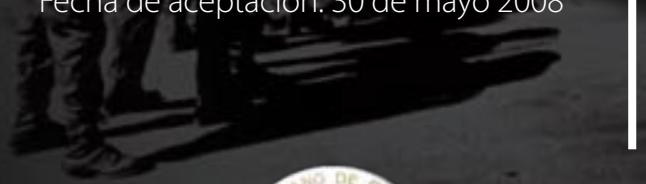

Una Teoría Alternativa De la Batalla de San Jacinto, 14 de Septiembre de 1856

Miembros del Consejo Editorial:

Dr. Ronny Viales, Dr. Juan José Marín

Editores Técnicos: 1

Allan Fonseca, Andrés Cruz, Gabriela Soto
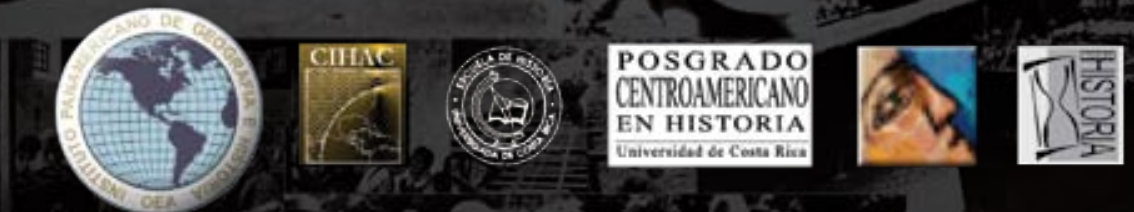
Indexaciones: Repositorio de Revistas UCR, DIALNET, Latindex, REDALYC Directorio y recolector de recursos digitales del Ministerio de Cultura de España, Directory of Open Access Journals. Diálogos Revista Electrónica de Historia ISSN 1409-469X. Número especial 2008. Dirección web: http://historia.fcs.ucr.ac.cr/dialogos.htm

\section{Una Teoría Alternativa De la Batalla de San Jacinto, 14 de Septiembre de} 1856

Pat Werner

Miembro Correspondiente Academia de Geografía e Historia de Nicaragua Ave Maria University Latin American Campus

San Marcos, Nicaragua werner@avemaria.edu.ni werner@ibw.com.ni 
En Nicaragua uno de los primeros elementos que los alumnos de la primaria aprenden muy temprano en sus estudios es el nombre de la Batalla de San Jacinto y su fecha, el 14 de Septiembre de 1856. Además, se habla de una batalla de cuatro horas de duración cuando las fuerzas del Coronel José Dolores Estrada fueron atacados por 200 filibusteros. Estrada, después de un largo combate, ganó.

Un día hace 14 años yo recorrí el campo de la batalla con el Dr. Alejandro Bolaños Geyer, médico e historiador que había pasado 25 años estudiando y recuperando la historia de Walker y su impacto en Nicaragua. Su trabajo rindió cinco tomos y miles de documentos y sin duda la base de datos más completa que ha existido o ya existe sobre el tema.

Después de leer los reportes de testigos oculares y conocer algo de las armas que los dos grupos usaban, llegué a una conclusión que la batalla duró mucho menos tiempo, tal vez 10 minutos o tal vez menos. Don Alejandro reía, y contestó que yo estaba fumando cigarrillos raros. Pero después de explicar mis inquietudes, no rió más.

\section{Sobre la Metodología:}

Como en otras obras, la idea es rechazar fuentes históricas, como comentarios años o décadas después escritos por personas no involucrado con los eventos, rechazar fuentes con un contenido fuerte de conclusiones e ideología, basar la historia en testigos oculares lo más posible, estudiar las fuentes contemporáneas oculares con mucha exactitud, y tomar en cuenta las niveles de tecnología disponibles en su época. Y finalmente, hay que pasar mucho tiempo andando por al sitio, con documentos, mapas, etc., y andar sobre cada centímetro del terreno y tratar de reconstruir en forma de video lo que pasó y lo que no pudo haber pasado.

1. Las Fuentes. Existen cinco fuentes importantes que ponen mucha luz en la batalla. A. El reporte de la Batalla escrito por el Coronel Estrada, el comandante de las fuerzas aliadas en la hacienda y escrito el mismo día de la batalla. B. el reporte de William 
Walker que publicó en su periódico, El Nicaragüense, la semana después de la batalla. También las referencias de Walker en su libro, The War In Nicaragua, escrito poco antes de su muerte en 1860, sigue sin cambio los datos publicados en El Nicaragüense. C. la información encontrada en el libro de Francisco Ortega Arancibia, alcalde de Masaya y soldado en la guerra Nacional. Publicó su libro 50 años después de la batalla y contiene una historial general de 1838 a 1878, pero él estaba en los cuarteles y otras batallas y escribió sobre los hechos con poca ideología. ${ }^{1} \quad$ D. Las Obras Históricas Completas, escrita por Jerónimo Pérez en 1865. Pérez fue político de Masaya y fue involucrado en todos los eventos políticos de Nicaragua de 1854 a 1868. Se casó con la hermana de Tomás Martínez, el ganador de la Guerra Nacional y dictador de 1858 a 1868 . Fue un apologista por Martínez, pero fue testigo ocular de muchos de los acontecimientos de la Guerra Nacional, incluyendo posiblemente siendo presente en la plaza de Masaya cuando Walker lo atacó en la batalla más grande y probablemente más importante de la Guerra Nacional. Cuando escribe prevaricaciones, y lo hace, es sobre Martínez y su política. En asunto de hechos de la Guerra Nacional algunas veces él toma los números e información directamente de Walker y lo cita, sin acusarlo de exageración o números falsificados. E. La Historia de Nicaragua, escrita por José Dolores Gámez en 1888. Contiene un trato con menos exactitud de los demás y no tiene datos oculares.

2. La Tecnología. Los autores mencionados describen al menos cinco tipos diferente de armas. Además el Dr. Bolaños había obtenido facturas de las arsenales de Gran Bretaña donde vendieron a Costa Rica cantidades impresionantes de fusiles y carabinas tipo Enfield, Modelo 1853, de calibre .577, con estrías (Comunicación personal de Alejandro Bolaños en 1994 y mostró al autor fotocopias de las facturas, con fecha de 1854.). Además el autor conoce de un rifle carabina, tipo Enfield M 1853, con proveniencia del campo de la batalla de San Jacinto. Las armas incluyen:

\section{De Los Filibusteros}

1 La critica más fuerte de la obra de Ortega Arancibia fue hecho por Alejandro Bolaños, nacido en Masaya, con familia en Masaya comenzando en el siglo XVIII, fue que Ortega fue tan completo en su descripción de la sociedad y personas en la Masaya de la Guerra Nacional que ni Bolaños podía entender de todos de las personas mencionado por Ortega. Bolaños encontró que todos las referencias, que él conocía, de personas, fechas, hechos, eran completamente confiable. Comunicación personal, 1992. 
Fusil rifle de US, modelo 1841, conocido el Mississippi Rifle, de calibre .54, con estrías. Mencionado por las fuerzas de Walter. Fue el rifle, tipo "Mississippi", de dos bandas, modelo 1841. De calibre 54, con una carga de 60 granos de pólvora, con una velocidad de 950 pies por segundo. El cañón tenía estrías y el proyectil era una pelota redonda de alrededor de 280 granos. Este fusil fue usado en la Guerra con México y tenía una puntería muy fina.

\section{Fusil rifle de US, Modelo 1855 Springfield, calibre .58 con estrías.}

La segunda arma usada en la batalla fue el fusil de minie (escrito minic por Ortega Arancibia). Este fue el fusil, modelo 1855, fue fabricado en Springfield, Massachussets la fábrica oficial del gobierno de los EEUU, y adoptado por el ejercito americano en 1855. Era de calibre 58, y tenía tres estrías, con una gira de 72 pulgadas, en su cañon para estabilizar el proyectil. Fue la primera arma diseñada para la bala Minie en los EEUU; antes los fusiles americanos con estrías usaban balas en forma de pelotas. La bala fue oblongada y pesaba 476 granos, con la base hueca, más o menos una copia de la bala Minie, inventado por el ejercito Francés en 1845. Tenía carga de 60 granos de pólvora y una velocidad en la boca del cañón de 900 pies por segundo. Su gran ventaja es que se podía tirar y recargarlo muy rápidamente, a pesar de las cenizas de la pólvora negra, que dejaba mucha suciedad dentro del cañón hasta que no se podía empujar otra bala por la garganta del cañón. La bala de este fusil minie era muy delgado y se entraba fácilmente. Los gases generados por la ignición de la pólvora negra pegaban duro a la base del proyectil y se abrió para agarrar las estrías del interior del cañón. Esto dio estabilidad y puntería a la bala.

Fusil carabina Enfield de Gran .Bretañia, de calibre .577 con cañón de 24 pulgadas y con estrías para usar bala tipo Minie. Utilizaba una carga de 60 granos de pólvora negra y la bala pesaba alrededor de 500 granos en forma alongada con la base hueca.

Todos estos fusiles se recargaban por la boca del cañón, y tenía un sistema de ignición de fulminante de mercurio, muy seguro. Su propellente fue pólvora negra. 


\section{Carabina Sharps, modelo 1853 "slant breech" (cerrojo inclinado)}

La cuarta arma usada en la batalla fue la carabina Sharps, modelo 1853, con cerrojo inclinado, de calibre 0.52 pulgadas. Fue el primer modelo del mecanismo Sharps vendido comercialmente. Poco después de la guerra nacional en 1859, la compañía Sharps cambió el diseño para que el cerrojo subiera y bajara verticalmente, no inclinado. Por eso, por su rareza (fabricaron un total de mil armas más o menos, muchas usadas en la guerra de guerrilla en Kansas en 1859 y nunca fue vendida internacionalmente) un rifle tipo Sharps, con cerrojo inclinado, encontrado en Centro America, probablemente fue utilizado por las fuerzas de Walker. Esta arma fue mencionada por Walker y fue cargado detrás del cañon de manera de un cerrojo que el tirador podía bajar muy rápido, recargar la recamara, subir y cerrar la recamara. Tenía cañón de 25 pulgadas y era de calibre 52, o sea el diámetro de su bala fue 0.52 pulgadas. Su cañón tenía estrías pero no se sabe el tipo de bala que usaba, pero la más común fue una bala alongada. Su ventaja era que se podía tirar y recargar más rápido que cualquier otro tipo de fusil. Fue utilizado por el ejercito americano en su guerra civil como arma de precisión de los francotiradores. Hay un ejemplar de este modelo en el museo militar del ejército de El Salvador (Walker: 299).

Revólver Colt, Modelo Primero de tipo Walker, 1847, calibre 0.44. Tal vez los más notables fueron los revólveres mencionados por ambos, Walker y Francisco Ortega Arancibia. Ortega Arancibia notó que cada revolver tenía un cañon de nueve pulgadas de largo. Este describe el revólver Colt Walker, primer modelo. Más tarde hubo un segundo y tercer modelo. Pero el primer modelo era el único Colt revólver que tenía un cañón de nueve pulgadas. Nombrado por un tal capitán Walker del ejército americano durante la Guerra con México (no era familiar de William Walker), la idea fue fabricar un revólver con tanto poder para matar a un jinete o a su caballo de un solo tiro. Y lo logró. Pesaba casi cinco libras con tambores vacíos y tenia casi 18 pulgadas de largo. Era de calibre 45 aunque lo llamaban de calibre 44, con diámetro de bala de 0.451 pulgadas. Tenía dos tipos de balas, uno la pelota de 145 granos, la otra bala en forma alongada con punta muy aguda. Su cargo de pólvora fue hasta 60 granos, la carga regular 
para fusiles. Fue el revólver más poderoso del siglo 19 y no fue alcanzado en poder hasta una invención del cartucho .357 magnum en 1935. Su bala de 200 granos tenía una velocidad de 1,100 pies por segundo. Fue muy útil para la caballería americana durante la Guerra con México y las tropas de Walker tenían el revólver más poderoso y peligroso de la época. Su tambor tenía recamaras para seis tiros, pero después el tirador tenía que desarmar el revólver para recargar el tambor, no con cartuchos, pero con pólvora y bala en frente y un fulminante detrás de cada recamara para encender la pólvora. (Walker:285; Ortega Arancibia: 329). Ortega Arancibia menciona que los aliados nunca habían visto armas como los revólveres Colt o fusiles tipo Minie y las armas encontradas en el campo de la batalla de San Jacinto eran una sorpresa.

\section{De Los Aliados:}

\section{a. Fusil, sin estrías, de sistema de ignición de chispa, de calibre aproximadamente, .69 , sin estrías, con bala esférica.}

Las armas del Coronel Estrada son más problemáticas. Se menciona que el gobierno del Presidente Carrera en Guatemala envió armas a los nicaragüenses. En otros documentos se habla de las armas de chispa, que hacían luz cerca de la cara del tirador cuando se disparaba. La invención del sistema de percusión, utilizó una copita de fulminante de mercurio para encender la pólvora, fue inventada en 1814 y la mayoría de los ejércitos en Europa y las Américas habían convertido los fusiles viejos a este sistema nuevo o habían adquiridos fusiles nuevos alrededor de los años 50. La gran ventaja de este sistema de ignición con fulminante de mercurio es que funcionaba más seguro, más rápido y era más fácil para entretener a un soldado campesino que el sistema de chispa que utilizaba un pie de gato con una piedra de pedernal para generar chispas al chocar con el rastrillo para encender la pólvora negra.

Las armas enviadas a Nicaragua por el Presidente Rafael Carrera de Guatemala, y después usados por las fuerzas nacionales en contra de los filibusteros, eran probablemente armas españolas que 
existían en las bodegas del ejercito después de la independencia. Las armas de España, después de 1700 y bajo el reinado de Felipe V, eran influenciadas por las armas de Francia. Se ve esta influencia en los fusiles del ejercito español. Comenzando en el modelo 1752 de fusil, eran de calibre 69. La llave del fusil tenía un diseño parecido a la del famoso fusil francés "Charleville" de 1769. El pie de gato y el rastrillo de la llave del fusil modelo 1791 y 1803 tenía más rasgos de influencia española, pero dentro del la forma del fusil Charleville. La desventaja del sistema de ignición de chispa era que se ensuciaba fácilmente con la pólvora negra y no encendía, le afectada mucho la humedad, hasta llegar al punto de no encender solamente por razón de la humedad en el aire (Brinckerhoff y Chamberlain:28-39).

Es muy probable que las armas utilizadas por el Coronel Estrada en la batalla de San Jacinto, el 14 de Septiembre de 1856, fueran de uno o más modelos de los fusiles españoles, del año 1752, 1791 ó 1803. Todos eran de calibre 69, con cañón sin estría o lisa, con una carga de pólvora negra alrededor de 60 granos, con un proyectil de pelota redonda, con un peso de 350 granos, más o menos, con un diámetro de 0.650 pulgadas aproximadamente, y con una velocidad de 800 pies por segundo.

Es posible que los protagonistas usaron más y diferentes tipos de armas, pero las armas notadas arribas son mencionadas en las obras citadas como las armas usados.

Las características de pólvora negra. La pólvora negra, inventado por los Chinos hace casi mil años, tiene varias características que define las armas que lo usan y varios límites de las armas. Su componente mayor es nitrato de potasio, un tipo de sal que es muy higroscopio y causa mucho sarro en el hierro muy rápido. Además, agrega mucha humedad de la atmósfera. Cuando ha absorbido demasiada humedad pierde su fuerza o no explota. Cuando explota, deja alrededor de 35-40 por ciento de su masa en el arma en forma de ceniza espesa, grasosa, sucia y mal oliente que entra en el mecanismo del arma y tapa su movimiento interno. A diferencia de la pólvora sin humo, inventada en 1885 y que no deja ceniza más que un vapor de agua y nunca explota, la pólvora negra explota cuando es confinado y en el abierto y genera una ola de choque con velocidad de 950 
p.p.s. La pólvora sin humo genera una ola de choque de varios miles de p.p.s y dinamita, el original explosivo sin humo genera una ola de choque de 10,000 p.p.s., sin ceniza.

El efecto de las características de las armas de pólvora negra fue que los fusiles acumulaban mucha ceniza en sus cañones y mecanismos después de dos o tres tiros y los ejércitos pusieron balas en sus cartuchos mucho menos del diámetro del cañón. Después de 50 metros no tenían puntería. Si hubiera mucha humedad en la atmósfera las cenizas podían impedir al tirador recargar su arma hasta que fuera limpiado el cañón con un cepillo o con agua. Por eso, la bala Minie fue inventado, fue mucho menos del diámetro del cañón. La base de la bala era hueca y abrió con la detonación de la pólvora. Las estrías causaron que la bala girara, lo que lo hizo muy estable en su vuelo.

El problema de las cenizas de la pólvora negra causó mucho más problemas con los revólveres Colt y sus mecanismos. Su primer revólver fue el modelo 1836, que fue muy delicado. Cuando los EEUU comenzaron a pelear en contra de México, el ejército pidió un arma corta con mucha fuerza que podía matar a un jinete o su corcel con un tiro. El Colt Modelo 1847 podía. Utilizaba una bala puntita de 145 granos de peso con una carga de hasta 60 granos de pólvora, la carga regular de fusiles. Era muy poderosa. Pero también tenia una falla de diseño: con cargos grandes de pólvora, después de tirar dos tambores completos de 12 tiros, las cenizas podían $\mathrm{y}^{2}$ muchas veces lograron parar el giro del tambor. La única manera de resolverlos fue desarmando el arma, limpiarla con un cepillo o agua, secarla y recargarla otra vez.

Otra característica de las armas de esta época es que usaban cartuchos, pero no cartuchos de metal, sino de papel o Lina saturado con nitrato de potasio. Para el Colt, aunque fue posible recargar el tambor sin sacar el cañón, era incomodo. Lo más rápido era sacar el cañón, poner seis cartuchos de Lina, abrirlas por detrás, reponer el tambor, reponer el cañón, poner los fulminantes detrás de cada recamara, y halar el gatillo. Para los fusiles,

2 En la juventud del autor, trabajó como armero en una armería en los EEUU. Tuvo la oportunidad de experimentar con muchas tipos de fusiles, pistolas y revólveres, incluyendo armas de pólvora negra. Tuvo dos Revólveres Colt, un tipo Walker y un tipo del ejército Modelo 1860. Los dos tenían el defecto de parar de operar después de 12-18 tiros con cargas máximas de pólvora. 
el proceso fue de morder la parte detrás del cartucho de Lina, usar el palo para forzarlo hasta la recamara del cañón, reponer el palo, montar un fulminante en el tubito cerca de la recamara, apuntar el fusil y apretar el gatillo. Con los fusiles de chispa, era más complicado. El tirador tenía que morder el cartucho detrás de la bala, poner un poco de la pólvora en el rastrillo, cerrar el rastrillo, poner el resto de la pólvora en el cañón, usar el palo para empujar el cartucho a la recamara, poner la pierna de gato detrás, apuntarlo y halar el gatillo.

Como descubrió Napoleón en Waterloo, un grupo de infantería muy bien entretenido en dos o tres filas puede continuar un fuego continuo y derrotar el mejor ejército del mundo, cada soldado tirando tres tiros por minuto. Pero con tropas de campesinos con armas de chispa, enfrentando soldados con armas lo más moderno, fue muy probable que pudieran hacer uno o no más de dos tiros con eficacia.

Con los tiradores de los revólveres Colt, podían tirar seis tiros casi instantemente, y con práctica y cartuchos disponibles de Lina tirar 12 tiros muy rápidamente, pero probablemente, después de 12 tiros, por razón de las cenizas y la suciedad de la pólvora negra, tenían que desarmar el revólver y limpiarlo antes de continuar el tiroteo.

\section{Los Reportes:}

Probablemente el reporte más importante es el reporte del Coronel José Dolores Estrada. Un natural de Nandaime, nació en el año 1792 y en la batalla tenía 64 años. Fue uno de lo más viejo peleadores que había tomado parte en varios de los alzamientos y revoluciones que sufría Nicaragua desde la independencia. Fue conservador, no con educación militar como el General Trinidad Muñoz, sino con mucha experiencia. Sabía de armas y las limitaciones de sus tropas.

Sobre el tamaño del ejército de Estrada, dijo que tenía 160 hombres. Estrada escribió que los filibusteros atacaron con una fuerza de 200 hombres. Después de la batalla reportó 
que perdió 55 hombres. Duración de la batalla reportada por Estrada: cuatro horas. Estrada reportó que en el campo de la batalla encontraron 22 revólveres, 32 fusiles y 47 cartuchos.

Hay otra observación de Pérez: que Estrada mandó, por las pocas municiones, que sus tropas solamente tiran a distancia de quema ropa.

Sobre la fuerza de Walker, se nota que compuesto de voluntarios, no regulares, y fue liderado por Byron Cole, el abogado de Nueva York que inicialmente interesó a Walker en Nicaragua. No tenía experiencia militar. En total las fuerzas de Cole numeraban 63 hombres (número aceptado por Bolaños). El número de muertos en la batalla reportado por Walker y Estrada fue de 27. Nadie de las fuerzas de Walker reportó la duración de la batalla.

Reportes de Bajas: El reporte de Estrada menciona detalles importantes. Nota que el fuego de los filibusteros fue muy nutritivo y que nadie podía sobrevivirlo. Notó también que los filibusteros atacaron a su lado izquierdo donde había corrales. Se nota que al frente de la hacienda y al lado izquierdo en el suelo hay piedra sólida, no tierra. Durante el ataque de los filibusteros es probable que los aliados recibieran sus bajas en hombres puestos en los corrales. Pérez reportó que Estrada tenía 55 bajas (Pérez: 270).

Descripción Del Campo de Batalla y La Hacienda San Jacinto. Ubicado 38 km al norte de Managua en un sector de producción ganadera, al este a dos km hay unas montañas. La hacienda esta situada al este-oeste, grande con cuatro salas y con paredes de adobe aproximadamente un metro de grueso. A la izquierda del portón principal había unos corrales probablemente de piedra. El terreno es plano. 


\section{Análisis de la batalla:}

Los reportes de Walker y Estrada son muy parecidos. Reportaron que las fuerzas de Walker salieron de Tipitapa, $12 \mathrm{~km}$ distante, y llegaron a la hacienda temprano la mañana del 14 de septiembre. Estrada no había puesto centinelas y había neblina. Los filibusteros podían acercarse a la hacienda. Los filibusteros se dividieron en tres bandas y atacaron a puntos diferentes. Walker pensaba usar el fuego de los revólveres Colt puso una sabana de fuego para aterrizar y derrotar a los aliados. Escribió:

"Cole halted a few minutes to arrange his plan of attack; and dividing his small force into three bodies, placed the first in charge of Robert Milligan, an ex-lieutenant of the army, the second under Major $\mathrm{O}^{\prime} \mathrm{Neal}$, and the third under Captain Watkins. The attack on the enemy was to be made at three points, and the weapons to be used principally were revolvers. These arrangements being made, the order to charge simultaneously the points assigned to each division was given. The order was gallantly obeyed, and Cole with Marshall and Milligan had already gained the corral when they were struck down with well-directed fire of the enemy. . . . Thus, almost at the same instant, and when the men were within a few rods of the house, all of the leaders and nearly one third of the whole force were either killed or wounded. Then the others, seeing nothing was to be accomplished with their numbers, withdrew, carrying off their wounded; and in a few minutes they were in full retreat toward Tipitapa." (Walker, 1860: 285).

El reporte de Estrada está de acuerdo:

"That they did, throwing their entire strength against our left wing, while at the same time launching hit and run attacks against our front. Although suffering heavy casualties, they took possession of part of the corral on our flank after they killed Don Ignacio Jarquín, heroic officer who held his post with honor and fought face to face with the enemy until he lost his life.

Others fell after Jarquín, for the enemy had gained a better position on the ground and 
their firm and sustained fire was doing great damage to our forces. Seeing that we could not regain that point by a frontal attack, because no man could possible advance through that barrage of bullets, I ordered Captain Liberato Cisne, and Lieutenants José Ciero and Juan Fonseca, to go out with their men on a flanking movement and attack the enemy from the left."(Bolaños: 277-278).

\section{Como dijo Pérez:}

"Estrada fue sorprendido; no tenía espionaje, y cuando le anunciaron al enemigo fue a corta distancia, porque la campaña era tan limpia, las brumas de la mañana impedían ver los objetos de lejos. Sin embargo, dio la orden de no dispararles, sino a quema ropa, sin duda para economizar el parque de que andaba tan escaso."(Pérez: 261).

Descripción de Estrada por Pérez: "Estrada fue militar desde su juventud; pero demasiado común en sus capacidades, debía los ascensos al valor y honradez, que poseía en alto grado. Así, era muy a propósito para ejecutar cualquiera operación; mas no para dirigirla. Era confiado por lo mismo que era valiente."(Pérez: 260).

\section{Reconstrucción De La Batalla del Autor:}

Cole y sus tropas llegaron a la hacienda San Jacinto a las 6:00 a.m. y se dividieron en tres grupos. Atacaron en tres sectores diferentes. A la izquierda por los corrales de piedra Estrada había puesto aproximadamente 50 soldados, con fusiles de chispa, dejando aproximadamente 100 soldados, con fusiles, dentro de la hacienda. Con las paredes gruesas, la gente dentro de la hacienda era protegida de todas las balas de los filibusteros. Si se supone que todos de los filibusteros tenían los Colt, sería 63 revólveres, de seis tiros, o 378 tiros disponibles en segundos. Los defensores tenían 50 tiros disponibles y después necesitaban al menos 30 segundos o más para recargar sus fusiles en tiempo de estrés de enemigos tratando de ventilarlos con balas Colt muy cercana. ${ }^{3} \mathrm{Si}$ los filibusteros

3 La tecnología de recargar los fusiles de chispa fue bien desarrollado y conocido. Estas armas eran idénticas con las armas usadas en las campañas Napoleónicas. Weller nota que la infantería de Gran Bretaña, lo mejor del mundo, podía tirar tres tiros por minuto, no más (Weller: 173-174). 
recargaban sus tambores una vez tendría 756 tiros disponibles en pocos minutos. Esta cantidad de tiros es probablemente lo que refirió a Estrada. Probablemente los filibusteros tenían éxito en matar o herir a la gran mayoría de aliados puestos en los corrales y por eso en pocos minutos los aliados perdieron cincuenta hombres.

Fue de gran importancia que Estrada no perdió su sentidos al ver un tercer de sus hombres asesinados en pocos minutos con armas que no habían vistos antes. (Pérez: 262; Ortega Arancibia: 137). Dio la orden de no tirar a distancia quema ropa, tal vez dentro de 30 metros. Los fusiles no tenían miras de ningún tipo y los soldados lo apuntaban como escopetas. El reporte de Walker habla de que los filibusteros llegaron a pocos metros, probablemente menos de 30 metros de la hacienda. Estrada ordenó a un grupo a salir detrás y atacar desde la izquierda, es decir probablemente poner a los filibusteros en un fuego enfilade, de frente y al lado. Y murieron tan rápido los filibusteros porque Estrada mandó a sus tropas a tirar en tiros unidos a una distancia probablemente menos de 30 metros. Es importante notar que todos los líderes de los tres grupos de filibusteros eran matados o heridos, probablemente en frente de sus tropas. Con este tiro andanada, la batalla terminó y los filibusteros, sin líderes, salieron hacia Tipitapa.

Si se compara el número de bajas de Estrada, 55 de 160 y 27 de 63 de Walker, usando las armas identificadas, se ve una batalla de pocos minutos, con los filibusteros contando en el fuego de sus revólveres para poner terror en los aliados. Los filibusteros no tenían liderazgo competente. Sin cañones para derribar las paredes de la hacienda, los filibusteros podían matar a la gente afuera pero no podían hacer nada con la gente dentro de la hacienda. Las paredes de la hacienda, de más de dos pies de grueso, completamente protegían a los aliados de las balas de Colt de los filibusteros. Todo lo que Estrada tenía que hacer era esperar que los filibusteros se acercaran al portón principal y hacer un tiro andanada, a distancia quema ropa, para acabar con los filibusteros cuando ellos estaban reunidos juntos, hombro a hombro. Y eso es lo que hizo. No perdió su sentido a pesar de que había visto en su vida el fuego más nutrido, y ganó. Aunque hasta hoy muchos han galardonado a Estrada por derrotar los filibusteros después de una batalla de cuatro horas, es probable, basado en su voluntad y habilidad, derrotó a los filibusteros en 10 minutos. 
Sobre el asunto de su reporte de un ataque de 200 soldados por cuatro horas, hay que tener respeto al Coronel Estrada. Un minuto bajo fuego parece como una hora. Y es muy posible que no quería, “contar narices” cuando habían docenas de balas llegando a los corrales y probablemente a todas las ventanas y portones de la hacienda.

También, se puede reconstruir el número de tiros que 200 soldados pudieron haber hecho: en el primer minuto al menos 1,200 tiros, y con tambores recargados, 2,400 tiros. Y no se sabe como pelearon los filibusteros con sus líderes muertos.

Finalmente, la determinación de lo que pasó en la batalla de San Jacinto no se va a finalizar con analices de reportes, pero si con la arqueología histórica del campo de batalla. Comenzando con investigaciones de la batalla de Waterloo (Keegan) y la batalla de George Custer del Little Bighorn (Fox), hay técnica de buscar en el suelo lo que pasó en una batalla, es una opción muy importante para clarificar realmente lo que pasó. Hoy día el campo de la batalla de San Jacinto es un monumento nacional. Actualmente el campo esta más o menos en la misma condición que el día de la batalla. Es el único campo de batalla en Nicaragua que esta intacto. Es posible que el departamento de arqueología haya encontrado la fosa común donde enterraron a los muertos. Tenemos los nombres de varios de los muertos, aliados y filibusteros y es posible contar el número de cadáveres en la fosa. Basado en los reportes deberían ser 78 ó 80 . Una investigación debe de determinarlo con exactitud.

Además, se puede reproducir los resultados asociados con las dos teorías de la batalla, teoría A, de una batalla de cuatro horas con un ataque de filibusteros de 200 hombres. La teoría B es una teoría de 63 filibusteros con batalla de 10 minutos. Y se tiene que ver que hay dos grupos de datos que existen en el sitio todavía, la fosa común de los muertos, ubicada por el departamento de arqueología del Museo Nacional de la República, y las 
paredes de la hacienda que recibían las balas de los filibusteros y donde casi seguro las balas se quedaron hasta hoy día. ${ }^{4}$

\section{Alternativa A: Batalla de cuatro horas:}

a. Número máximo de revólveres Colt, 200.

b. Número máximo de tiros en los primeros dos minutos: 2400, dirigido a los corrales y las paredes de la hacienda. En cuatro horas hay tiempo para limpiar el mecanismo recargar los tambores y tirar al menos tres tambores, o 18 tiros, a la velocidad de una vez por hora ó 3,600 tiros más, por un total de 6,000 tiros.

c. Número máximo de tiros en los cuerpos de los aliados: 55 o tal vez 80, contando con la mitad de los aliados recibiendo dos tiros. Todos con balas con estrías de calibre .44 con diámetro de 0.451 pulgadas en forma de pelotas o conos truncados.

d. Número máximo de tiros en los cuerpos de los filibusteros: 27, o tal vez 42 contando con la mitad de los filibusteros recibiendo dos tiros. Todos con balas sin estrías de calibre 0.65 en forma de pelotas.

e. Número máximo de balas que pegaban en las paredes de la hacienda: difícil para calcular, pero si se percibe que todos los filibusteros tiraban generalmente en la dirección de los corrales y la hacienda y sus paredes, se piensa tal vez en un cuarto de las balas tiradas, ó 1,500 concentrados por las ventanas y portones principales, y probablemente se pueden detectar por los zapadores del Ejercito de Nicaragua.

f. En la fosa común, alrededor de 55 balas de tipo Colt y 27 de tipo de fusil de calibre 0.65 .

\section{Alternativa B: Batalla de 10 minutos:}

a. Número máximo de revólveres Colt, 63.

4 La suposición es que las paredes de hoy día de la hacienda son las mismas del día de la batalla con una excepción. En 1994 el autor entrevistó el señor Knoepfler, cuya familia fue el dueño de la hacienda al comienzo de los años 60. Dijo que la hacienda fue declarado utilidad pública y confiscada alrededor de 1964 por el Presidente Luís Somoza. Pero el gobierno nunca pagó por la hacienda. Además, un parte, del lado sur de la hacienda fue reparado porque estaba cayendo al suelo. Por eso probablemente este parte de la hacienda no contendría nada de balas. Pero los demás, sí. 
b. Número máximo de tiros en los primeros dos minutos: 756, contando en tirar dos tambores completos, dirigido a los corrales y las paredes de la hacienda. c. Número máximo de tiros en los cuerpos de los aliados: 55 o tal vez 80, contando con la mitad de los aliados recibiendo dos tiros. Todos con balas con estrías de calibre .44 con diámetro de 0.451 en forma de pelotas o truncadas.

c. Número máximo de tiros en los cuerpos de los filibusteros: 27 , o tal vez 42 contando con la mitad de los filibusteros recibiendo dos tiros. Todos con balas sin estrías de calibre 0.65 en forma de pelotas.

d. Número máximo de balas que pegaban en las paredes de la hacienda: difícil para calcular, pero si se percibe que todos los filibusteros tiraban generalmente en la dirección de los corrales y la hacienda y sus paredes, se piensa que tal vez un cuarto de las balas tiradas, o 189 balas, concentrados por las ventanas y portones principales, y probablemente se puede detectar por los zapadores del Ejercito de Nicaragua.

e. En la fosa común, alrededor de 55 balas de tipo Colt y 27 de tipo de fusil de calibre 0.65 .

Conociendo el tipo de armas usadas se puede verificar las balas de las armas, las de los filibusteros con estrías y las balas de Estrada probablemente lisas. Además es posible identificar armas individuales por las estrías y si hay hoyos en huesos, cuales balas los mató. Si hay calaveras es posible identificar si fueron mestizos o Europeos. Con detectores de metal se puede buscar más balas en la fosa y también registrar si hay pelotas de plomo en las paredes de la hacienda y en cual paredes y su número. Y este debe definir finalmente lo que pasó en San Jacinto el 14 de Septiembre de 1856. Porque al fin, lo que se encuentra en el suelo no puede contradecir lo que esta escrito por testigos oculares que escribían lo que veían. Vamos a ver los que se encuentra en el suelo y las paredes de la hacienda.

Finalmente, vale la pena considerar como la leyenda de San Jacinto creció. En analizar todas las batallas de la Guerra Nacional, se ve las tres batallas de Rivas y su estado de sitio en 1857, y las dos batallas de Masaya, octubre y noviembre, en 1856 eran batallas con mucho más participantes y con más importancia militar. Patricia Fumero, 
en particular, lo ha examinado y concluye que fue un atento de unificar la independencia simbólicamente con la batalla de san jacinto Aunque eran de partidos opuestos, Máximo Jerez era amigo de Estrada y su aliado en la Guerra Civil de 1862 en contra el Presidente Tomas Martínez. Después de la muerte de Estrada en 1869, Jerez y otros comenzaron a celebrar la Batalla de San Jacinto en honor de la memoria de Estrada, y así comenzó la tradición de conmemorar la batalla de San Jacinto ${ }^{5}$.

\section{Bibliografía}

Bolaños Geyer, Alejandro. William Walker. The Gray Eyed Man of Destiny. Cinco tomos. Privately Printed, St Charles, Missouri. 1991.

Brinckerhoff, Sidney and Chamberlain, Pierce, A. Spanish Military Weapons in Colonial America, 1700-1821. Stackpole Books, 1972.

Fox, Richard Allan Jr. Archaeology, History, and Custer's Laast Battle. University of Oklahoma, Norman. 1993.

Fumero Vargas, Patricia. “De La Iniativa Individual a la Cultura Oficial: El Caso del General José Dolores Estrada, Nicaragua Década de 1870“”, en Kinloch, Tijerino, Frances, Nicaragua En Busca de su Identidad, INHCA, Managua, 1995.

Gamez José Dolores. Historia de Nicaragua. Colección Cultural, BANIC, Managua, 1992.

Keegan, John. The Face of Battle, Guildford, Biddles. 1978.

Ortega Arancibia, Francisco. Cuarenta Años (1838-1878) de Historia de Nicaragua. Colección Cultural, Banco de America, Managua, 1975.

Pérez, Jerónimo. Obras, Completas. Colección Cultural, Banic, Managua, 1992.

Salvatierra, Sofonías. La Guerra Nacional. Adilá editor, Managua, 2006.

Sharpe, Philip. B. The Rifle In America. National Rifle Association, 1995.

Walker, William. The War In Nicaragua. University of Arizona Press, Tucson, 1985.

Weller, Jac. Wellington at Waterloo. Greenhill Books, London, 1992.

Young. " "Since Herodotus Has History Been a Valid Concept?" American Antiquity 53: 7-12. 1988.

$5 \quad$ Véase, “De la Iniciativa Individual a la Cultura Oficial: El Caso del General José Dolores Estrada Nicaragua, Década de $1870 “$ en, Nicaragua en busca de su Identidad, Frances Kinloch Tiberino, editor, 1995. 